\title{
SENTIMENTOS DA FAMÍLIA NA INTERAÇÃO COM A EQUIPE DE ENFERMAGEM
}

Teresa Cristina Gioia Schimidt ${ }^{1}$, Marlucy Lima de Arruda ${ }^{2}$

RESUMO: Pesquisa transversal, de campo e qualitativa realizada entre agosto e setembro de 2011 com 30 familiares que vivenciavam a experiência de ser acompanhante em hospital, com o objetivo de verificar seus sentimentos na interação com a enfermagem, identificando pontos de interferência, expectativas e sugestões. Os resultados quantitativos da entrevista foram analisados com auxílio de estatística descritiva e os qualitativos analisados pelo método de análise de conteúdo de Bardin, resultando nas categorias Acolhimento e Comunicação eficiente-eficaz. Os pontos positivos foram relacionados à ação técnica e comportamento; os negativos, às condições administrativas e confusão do papel do acompanhante. As expectativas foram atendidas, apontando para percepção positiva e satisfatória da qualidade da assistência; as sugestões valorizaram os aspectos operacionais e interacionais. Concluiu-se que a percepção do acompanhante sobre a qualidade do atendimento prestado pela equipe de enfermagem foi boa, sendo os aspectos relacionais, os mais citados.

PALAVRAS-CHAVE: Cuidados de enfermagem; Enfermagem familiar; Avaliação em saúde; Pesquisa qualitativa.

\section{THE FAMILY'S FEELINGS IN INTERACTION WITH THE NURSING TEAM}

ABSTRACT: This qualitative cross-sectional field study, carried out between August and September 2011 with 30 family members who had been through the experience of being a companion in hospital, aimed to ascertain their feelings in the interaction with nursing, identifying points of interference, expectations and suggestions. The quantitative results from the interviews were analyzed with the help of descriptive statistics, while the qualitative results were analyzed through Bardin's content analysis, resulting in the categories Welcoming and efficient-efficacious Communication. The positive points were related to behavior and technical action: the negative, to administrative conditions and confusion about the role of the companion. The expectations were met, pointing to the positive, satisfactory perception of the quality of the care; the suggestions valued the operational and interactional aspects. It was concluded that the perception of the companion about the quality of care provided by the nursing team was good, with the relational aspects being cited most.

KEYWORDS: Nursing care; Family nursing; Health evaluation; Qualitative research.

\section{SENTIMIENTOS DE LA FAMILIA EN LA INTERACCIÓN CON EL EQUIPO DE ENFERMERÍA}

RESUMEN: Investigación transversal, de campo y cualitativa realizada entre agosto y septiembre de 2011 con 30 familiares que tuvieron la experiencia de ser acompañante en hospital, con el objetivo de verificar sus sentimientos en la interacción con la enfermería, identificando puntos de interferencia, expectativas y sugerencias. Los resultados cuantitativos de la entrevista fueron analizados con ayuda de estadística descriptiva y los cualitativos analizados por el método de análisis de contenido de Bardin, resultando en las categorías Acogimiento y Comunicación eficiente-eficaz. Los puntos positivos fueron relacionados a la acción técnica y comportamiento; los negativos, a las condiciones administrativas y confusión del papel del acompañante. Las expectativas fueron atendidas, apuntando para percepción positiva y satisfactoria de la cualidade de la asistencia; las sugerencias valoraron los aspectos operacionales e interaccionales. Se concluye que la percepción del acompañante acerca de la cualidade del atendimiento prestado por el equipo de enfermería fue buena, siendo los aspectos relacionales los más referidos. PALABRAS CLAVE: Cuidados de enfermería; Enfermería familiar; Evaluación en salud; Investigación cualitativa.

\footnotetext{
${ }^{1}$ Enfermeira da Secretaria de Estado da Saúde de São Paulo. Doutora em Ciências. Professora Colaboradora no Departamento MédicoCirúrgico da Escola de Enfermagem da Universidade de São Paulo - EEUSP. Professora do Departamento de Ciências da Saúde da Universidade Nove de Julho - SP. Membro do Grupo de Pesquisa e Estudo sobre Comunicação em Enfermagem.

${ }^{2}$ Enfermeira.
}

Autor correspondente:

Teresa Cristina Gioia Schimidt

Universidade de São Paulo

Av. Dr. Enéas de Aguiar Carvalho, 188 - 05403-000 - São Paulo-SP-Brasil

E-mail: teresa.schimidt@gmail.com
Recebido: 06/11/2011

Aprovado: 22/03/2012

Cogitare Enferm. 2012 Abr/Jun; 17(2):348-54 


\section{INTRODUÇÃO}

A Enfermagem é uma ciência que estuda o cuidado prestado ao ser humano doente ou sadio, de forma individual, familiar ou integrada à comunidade. Sabidamente o cuidar é sua base, pois esta ação envolve não apenas procedimentos técnicos, mas também ligações emocionais, e ao realizar este ato estabelecem-se vínculo entre profissionais e clientes, incluindo os familiares. Considerando que os procedimentos do cuidar são, em sua maioria, especificamente ligados ao cuidado instrumental, há uma interferência na intimidade estabelecida. Por esse motivo, é preciso estar atento às respostas físicas e psicológicas expressadas pelo cliente, que as manifesta de modo verbal como não verbal ${ }^{(1)}$.

Pesquisa revela não ser costume da equipe de enfermagem incluir os familiares e acompanhantes durante os cuidados prestados ao cliente, um dos motivos está relacionado ao descaso e desconhecimento da importância de tal acolhimento ${ }^{(2)}$. Vale destacar que o conceito de família é definido não apenas por ligações genéticas, mas também por fatores emocionais que cerceiam os indivíduos. Por meio dela, desenvolve-se um indivíduo capaz de conhecer e valorizar os aspectos inerentes do convívio na sociedade na qual está inserido, isto porque sabe-se que cada sociedade defende valores éticos e morais distintos.

A estrutura familiar vem sofrendo modificações em sua composição: pai, mãe e filho que, por muitos anos, foram retratos familiares; atualmente dois novos grupos têm surgido, as famílias monoparentais e as recompostas. A primeira corresponde a situações em que o núcleo familiar é constituído por apenas um adulto e uma criança, e a segunda refere-se às formadas por laços conjugais após divórcio ${ }^{(3)}$.

A Enfermagem, independente do local de atendimento ao cliente, precisa valorizar e reconhecer a importância da família, pois todo indivíduo faz parte de uma conjuntura que foi modificada por algum motivo externo e que pode determinar repercussões não favoráveis à saúde individual, familiar e coletiva.

Na internação hospitalar, muitas vezes, tem-se uma visão distorcida relacionada às necessidades de procedimentos hospitalares, pois em algumas ocasiões o risco de morte é iminente. Ou ainda, quando há uma situação não controlada e eletiva, que provoca a internação, fato que causa ao cliente certa apreensão e medo, contribuindo para que reaja de maneira negativa ao tratamento proposto, tardando sua recuperação. Esse fato acaba por refletir nos familiares, deixando-os mais apreensivos e temerosos quanto aos cuidados a serem realizados pela equipe ${ }^{(4)}$.
Nesse contexto, familiares entendem que quando alguém amado está em dificuldade, precisam de ajuda para cuida/salvar seu ente querido. À equipe de saúde é a quem recorrem, quando fragilizados pelo medo de uma possível perda e pela aflição de ver o familiar em situação delicada, tornando a aproximação da enfermagem uma tarefa árdua. Torna-se comum os familiares compartilharem suas ansiedades e medos, eles almejam dos profissionais de saúde um tratamento atencioso, seguro e que revele preocupação ao cliente debilitado. Muitas vezes, uma simples conversa, um olhar ou mesmo a escuta silenciosa propiciam sanar dúvidas e, essa atitude, contribui na recuperação do paciente ${ }^{(5)}$.

Mas, infelizmente um grande número de hospitalizações provoca traumas a clientes e familiares. Ao familiar, em algumas ocasiões, é negado o direito de permanecer com o cliente pelo tempo desejado, mesmo sendo esse um direito assegurado. Outra situação negativa é revelada quando questionam os profissionais sobre o estado de saúde de seu familiar e recebem variadas respostas. Alguns respondem que não estão interados sobre o caso, outros que aquele questionamento só o médico poderá responder. Enfim, diversas respostas, mas nenhuma que provoque a tranquilidade do familiar ${ }^{(5)}$.

Muitas vezes, a equipe de enfermagem vê os familiares como objetos passivos que devem auxiliar em tarefas apenas quando lhes for permitido. Porém, o ideal seria que compreendessem que os familiares devem ser integrados ao tratamento, explicando cada procedimento a ser efetuado e mostrando como ele pode ajudar, pois é com a sua participação que se determina melhor aceitação ao tratamento e recuperação do cliente ${ }^{(6)}$.

Mesmo de forma imperceptível, a equipe de enfermagem é avaliada constantemente dentro do hospital pelos clientes e familiares. Esta razão possa ser um dos motivos que leva o profissional a manter um contato estritamente necessário. Alguns funcionários que integram a equipe de enfermagem entendem que o cuidado vai além da técnica e que faz parte dele o relacional e conseguem demonstrar alegria, bom humor e satisfação ${ }^{(7)}$.

Compreendemos que a inclusão da família no tratamento é de fundamental importância para a recuperação do cliente e, por intermédio desta pesquisa, almejamos saber: qual a percepção dos familiares e acompanhantes sobre sua presença e participação durante os cuidados de enfermagem?

Dessa maneira os objetivos desta pesquisa são verificar os sentimentos da família na interação com a equipe de enfermagem durante a internação de um ente querido; relacionar os pontos positivos e negativos 
da relação vivida; identificar se as expectativas frente ao atendimento prestado pela equipe de enfermagem foram atingidas e citar as sugestões para a melhoria da assistência prestada na instituição da pesquisa.

\section{MÉTODO}

Pesquisa de campo, transversal com abordagem qualitativa desenvolvida com 30 familiares que vivenciavam ou vivenciaram, nos últimos 12 meses, a experiência de ser acompanhante de um ente querido, quando internado nas unidades de clínica médica e/ou cirúrgica de um hospital público da capital paulista. A pesquisa foi iniciada após aprovação do Comitê de Ética em Pesquisa com Seres Humanos, sob Parecer COEP n. 0207/11.

Os critérios de inclusão dos participantes foram: Familiar, independente de sexo, com idade igual ou superior a 18 anos, que tenha permanecido/permanece como acompanhante de alguém de maior vínculo, independente da idade, e que tenha ficado/estivesse internado em qualquer tipo de unidade clínica e/ou cirúrgica por, pelo menos, 72 horas.

Os participante responderam um questionário composto de perguntas para a sua caracterização (sexo, idade, anos de estudo, grau de parentesco, número de dias de acompanhamento, número de vezes no último ano que vivenciou ser acompanhante daquele cliente e conhecimento do problema ou patologia que gerou a internação do cliente) e a cinco perguntas abertas pertinentes e em conformidade com os objetivos da pesquisa, a saber: 1) Descreva com suas palavras como você se sente/sentiu ao receber o atendimento da equipe de enfermagem; 2) Cite dois pontos positivos da relação vivida entre você e a equipe de enfermagem; 3) Cite dois pontos negativos da relação vivida entre você e a equipe de enfermagem; 4) $\mathrm{O}$ que foi oferecido pela equipe de enfermagem atendeu suas expectativas? e 5) O que poderia ser feito para que a assistência de enfermagem dada a você como acompanhante fosse melhorada? Todos os entrevistados foram identificados com a palavra "Familiar" seguida pelo número de ordem sequencial de entrevista.

A pesquisa foi aplicada entre agosto e setembro de 2011, tendo sido observados os preceitos éticos de sigilo, privacidade e confidencialidade. Não houve gravação das falas, o registro das informações se deu por meio de anotações e, ao término da entrevista, a pesquisadora mostrou para cada participante os relatos efetuados, fazendo acréscimos e acertos no que foi informado, validando assim o conteúdo coletado.

Os dados de caracterização foram digitados em planilha do programa Excel versão 2007, que serviu como banco de dados da pesquisa, facilitando a tabulação e a análise dos dados. O tratamento dos dados qualitativos foi realizado por meio de interpretação dos depoimentos, com base no método de Análise de Conteúdo de Bardin ${ }^{(8)}$. Este método é a aplicação de um conjunto de técnicas de análise da comunicação, que visa a obter por procedimentos sistemáticos e objetivos de descrição do conteúdo das mensagens, indicadores que permitam a inferência das percepções, expectativas e sugestões sobre o relacionamento dos familiares com a equipe de enfermagem no âmbito hospitalar ${ }^{(8)}$.

\section{RESULTADOS}

Dos 30 participantes, $77 \%$ eram mulheres, $66,7 \%$ tinham mais de 12 anos de estudo, $66,7 \%$ eram filhos ou filhas dos clientes internados, $16,7 \%$ esposas, $6,7 \%$ irmãs e $10 \%$ genros, noras ou sobrinhos; a idade média foi de 49,5 anos. O número médio de dias de acompanhamento do familiar foi de 11 dias, sendo o menor tempo de 3 e o maior de 46 dias.

Quanto ao número de vezes, que o familiar vivenciou o processo de internação com o mesmo cliente, foram duas vezes no último ano, 53\% acompanharam apenas a internação atual, $43 \%$ acompanharam de duas a cinco internações e 3\% acompanharam mais de seis internações. Quando questionados sobre as patologias que levaram à internação, $20 \%$ não souberam responder, dos que souberam, as patologias mais representativas, na ordem decrescente, foram: $37,5 \%$ doenças cardiorrespiratórias; $25 \%$ doenças neuroendócrinas; $16,7 \%$ doenças infecciosas; $8,3 \%$ doenças renais; $8,3 \%$ doenças hematológicas e $4,2 \%$ oncológicas.

Com base na análise dos conteúdos dos discursos dos familiares, o sentimento ao receber atendimento da equipe de enfermagem, deu origem a duas categorias: Acolhimento e Comunicação eficiente e eficaz.

A primeira categoria, denominada de Acolhimento, abrangeu a importância dada ao se sentir bem atendido e acolhido, ter recebido atendimento pontual e educado. Eis dois discursos que ilustram:

Fui sempre bem atendida e com educação. (Familiar 2)

Eu senti bem acolhida, bem atendida, todos foram atenciosos comigo. (Familiar 8)

$\mathrm{Na}$ categoria Comunicação eficiente e eficaz, a ênfase foi dada à promoção do cuidado, bem-estar, 
respostas aos interesses e orientações para a participação e/ou compreensão do que ocorre com o cliente, de acordo com alguns depoimentos:

\section{Eu percebi interesse por mim. (Familiar 9)}

Recebi as informações necessárias de forma que me sinto bem. (Familiar 30)

Vale informar que $50 \%$ dos entrevistados relataram ter recebido um bom atendimento e os outros $50 \%$ sentiram-se extremamente bem atendidos.

Quando os acompanhantes foram questionados sobre os pontos positivos da relação vivida entre ele e equipe de enfermagem, os resultados puderam ser categorizados em três categorias distintas: Ação técnica, Ação comportamental e Ação mista.

A Ação técnica (3,4\%- 1 familiar) teve sua composição dada pelas respostas fornecidas que caracterizavam estritamente a operacionalização do cuidado/ atendimento, ligado, basicamente, à pontualidade, pronto-atendimento e aplicação de princípios científicos. Esses garantiam segurança e revelaram profissionalismo, comprometimento e, ainda, forneciam informação técnica e resposta precisa às dúvidas.

A Ação comportamental (43,4\% - 13 familiares) foi composta pelas afirmativas com teor estritamente atitudinal do profissional de enfermagem, como atenção, interesse, dedicação, forma de dialogar e receber opiniões, inclusive divergentes, carinho, afeto, empatia, alegria, cordialidade, solicitude, bom humor, descontração e boa vontade.

A Ação mista (53,2\% - 16 familiares) reuniu as duas categorias anteriores, isto é, foram citações que abarcaram as duas condições como positivas: técnica e comportamental.

Em relação aos pontos negativos da relação vivida entre ele (acompanhante/familiar) e equipe de enfermagem, os resultados foram categorizados em Condição administrativa (75\% - 6 familiares) e Confusão do papel de acompanhante (25\% - 2 familiares). Vale ressaltar que $26,7 \%$ citaram algum ponto negativo, os demais alegaram não ter.

A condição administrativa reuniu as respostas com os depoimentos ligados à organização da unidade, número reduzido de pessoal no fim de semana, o que, na visão dos participantes, dificulta o relacionamento, o cuidado com o cliente e a demora no atendimento propriamente dito.

A confusão do papel do acompanhante englobou as falas que mencionaram crítica frente à compreensão equivocada do acompanhante durante a internação, pois como revela o familiar:

\section{Me criticaram por eu estar dormindo. (Familiar 11)}

Expectativas e sugestões foram levantadas no sentido de conhecer quais os desejos e esperanças que o acompanhante/familiar do cliente hospitalizado possuía em relação à hospitalização e ao cuidado propriamente dito. Todos foram unânimes em afirmar que as expectativas foram atendidas, o que aponta para uma avaliação da percepção positiva e satisfatória da qualidade da assistência de enfermagem neste aspecto. Os resultados revelaram que dedicação, profissionalismo, atenção, esforço, mesmo com as limitações, muitas vezes foram os itens considerados de maior evidência e que ratificam o atendimento das expectativas e a valorização do relacionamento interpessoal.

Constata-se que $50 \%$ dos pesquisados não conseguiram emitir sugestões, pois se sentiam satisfeitos com o atendimento recebido pela equipe de enfermagem. Os demais 50\% listaram sugestões; com a finalidade de facilitar a exposição das opiniões emitidas e as sugestões, foram agrupadas em duas categorias, a saber: as que Valorizam os aspectos operacionais (73,3\% - 11 familiares), composta pelas revindicações ligadas à ampliação do quadro funcional de enfermagem, transmissão de informação e notícias as condições do cliente via telefone, agilização na realização de exames requeridos e melhoria nas acomodações do acompanhante. Já a segunda categoria intitulada como as que Valorizam os aspectos interacionais $(26,7 \%$ - 4 familiares) reuniu as respostas que solicitavam que os funcionários continuassem sendo o que são e maior agilidade no atendimento das campainhas e reivindicam apoio psicológico para eles.

\section{DISCUSSÃO}

Conforme estudos desenvolvidos, a maioria dos acompanhantes não é capaz de avaliar a competência técnica dos profissionais de saúde e, por isso, avaliaramnos como seres humanos. Disso pode-se ressaltar a importância do cuidado humanizado, percebido pela linguagem não verbal ${ }^{(9)}$. Além disso, sabe-se que o cuidado humanizado envolve o respeito à individualidade do ser humano, e isso foi altamente valorizado nesse estudo.

Pesquisa realizada nos Estados Unidos da América categorizou o cuidado em dois grupos - o instrumental, que corresponde ao que, neste estudo, é a ideia central 
ação técnica e o expressivo, aqui categorizado como ação comportamental. O resultado do referido estudo mostrou que os norte-americanos reconheceram e valorizaram muito mais o cuidado instrumental (técnico) do que o cuidado expressivo (comportamental) ${ }^{(10)}$, o que vem de encontro aos resultados da presente pesquisa, talvez, pela especificidade cultural, pois os brasileiros valorizam muito a área comportamental (expressiva) ${ }^{(11)}$.

Em contrapartida, essa percepção vai ao encontro do verificado em pesquisa desenvolvida com idosos hospitalizados, revelando que $76,7 \%$ valorizaram o cuidado como significado da interação comportamental e os demais $(27,4 \%)$ detiveram-se em dar significado ao cuidado como ação com foco técnico ${ }^{(12)}$. O mesmo ocorreu em estudo realizado com cuidadores que estavam acompanhando seus entes em unidades de internação ${ }^{(11)}$.

Quando os familiares recordaram que a condição administrativa, a exemplo da organização da unidade, como um ponto negativo da relação vivida, isso remete à afirmação de que a qualidade das acomodações de um hospital, e seu conforto, merecem atenção especial, desde a escolha da cor das paredes, ventilação e iluminação das áreas até outros itens como a organização física do mobiliário existente ${ }^{(12)}$.

Outro ponto de destaque negativo apontado foi o número reduzido de pessoal nos fins de semana. Pesquisadores reforçam o fato de que, para fornecer qualidade de assistência, é necessário ter quantidade e nível adequado do pessoal de enfermagem nas unidades. Entretanto, alguns hospitais não consideram esse quesito essencial e acabam por se avaliarem bons baseando-se em outros parâmetros ${ }^{(13-14)}$. Adicionalmente, o fato de as metas, consideradas pelos dirigentes públicos da saúde no Brasil serem voltadas à produtividade, o quantitativo de pessoal não tem sido uma prioridade e acaba sofrendo cortes para o ajuste do orçamento o que, de uma forma direta, atinge a qualidade do serviço ${ }^{(15)}$.

A confusão do papel a ser exercido pelo acompanhante foi uma realidade deste trabalho e isso vai ao encontro de trabalho desenvolvido com a equipe de enfermagem em que se verificou que, muitas vezes, a responsabilidade do cuidado é nele totalmente depositada e cobrada pela equipe de enfermagem. Alguns dos acompanhantes assumem parte dos cuidados e isso, ao invés de ser encarado como oportunidade de ensino e orientação, ou ser interpretado pela enfermagem como uma forma de revelar zelo, atenção e até mesmo comprometimento com o cuidado de seu ente, é simplesmente analisado como uma obrigação, o que constitui um equívoco. É importante o reconhecimento da singularidade dos acompanhantes pelos profissionais de enfermagem, para que possam compreender suas idiossincrasias e, assim, devem ser inseridos de maneira adequada e ajustada no cuidado prestado, devendo ainda ser compreendidos quanto a seu papel, limites e possibilidades ${ }^{(16)}$.

É oportuno salientar que os profissionais precisam entender que os acompanhantes têm necessidades físicas de repouso, de alimentação e apoio emocional, pois assim poderão estar melhor preparados para se inserir no cuidado durante o processo de hospitalização ${ }^{(12)}$.

As sugestões apontadas e contidas nas opiniões aqui explicitadas foram ratificadas na pesquisa desenvolvida no interior paulista onde aspectos administrativos foram recordados e citados nas sugestões como uma forma que poderia ajudar os clientes a se sentirem mais confiantes e tranquilos ${ }^{(12)}$.

A solicitação para ampliação do quadro de pessoal para execução das atividades de enfermagem foi uma das sugestões citadas que se repetiu nos discursos. É sabido que a contratação de pessoal baseia-se na política institucional, no modelo de gestão, quantitativo de leito operacional, tipo de serviço oferecido, mas não se restringe a esses critérios. Sofre influência do conceito que a direção de enfermagem possui sobre o serviço, bem como os planos e metas que se deseja cumprir(17). É preciso ficar atento que o dimensionamento inadequado de recursos humanos em enfermagem traz implicações sobre o resultado da qualidade da assistência, em virtude dos aspectos quantitativos e qualitativos de pessoal estarem diretamente ligados ao produto final de seu trabalho, que é a qualidade da assistência prestada ao cliente ${ }^{(17)}$. Portanto, a sugestão de maior número de pessoal de enfermagem tem pertinência com o que se verifica na literatura.

Já em relação à transmissão de informações, estudo considera que a pessoa, quando internada, encontra-se num evento de crise e essa situação se estende à família e, muitas vezes, interfere na sua compreensão. Sendo assim, a equipe de enfermagem deve atentar às necessidades físicas e psicológicas de ambas e incorporá-las nas necessidades levantadas, e fornecer orientações e informações de forma efetiva. A informação oferecida ao familiar é muito importante, sobretudo para conhecer o motivo da internação, o que se faz para os clientes internados e como é a dinâmica do trabalho dos funcionários da unidade. $\mathrm{O}$ familiar 
precisa estar seguro de que a pessoa internada receberá toda a assistência de que necessita. Por outro lado, os funcionários parecem não saber como poderão transmitir essa segurança, tanto no que se refere ao tipo de informação que poderá ser dada quanto à interpretação dos cuidados prestados ${ }^{(18)}$. Outros profissionais justificam essa dificuldade pelo excesso de trabalho e alegam falta de tempo ${ }^{(19)}$.

Embora seja comum que os familiares tenham acesso à visitas constantes e recebam um boletim informativo onde está descrito o estado de saúde do cliente internado, eles desejam informações adicionais por meio de telefonemas, procurando maiores esclarecimentos sobre o que acontece ou o que foi transmitido. Ressalta-se que informativos por telefone não necessariamente garantem ser uma estratégia eficaz e que ofereça a segurança desejada, até porque se sabe que o Conselho Federal de Medicina proíbe essa prática, seja por telefone seja por meio eletrônico. Outro ponto relevante a ser ratificado é que a maioria dos familiares desconhece o significado dos termos técnicos empregados, sugerindo que as expressões contidas no informativo, além de dificultarem o entendimento, podem gerar momentos estressantes e angustiantes aos familiares e, ainda, podem favorecer interpretações e obter conclusões equivocadas sobre as informações recebidas ${ }^{(20)}$.

A segunda categoria de sugestões que foram aquelas que valorizam os aspectos interacionais são congruentes ao fato de que atender o cliente com atenção e rapidez constitui-se em um dos quesitos importantes para o cuidado/tratamento de qualidade ${ }^{(17)}$. Outro aspecto relevante é compreender que o comportamento do cliente, quando hospitalizado, pode sofrer alterações como desorientação, não aceitação do tratamento e dos cuidados, entre outros ${ }^{(12)}$. Muitas vezes, a equipe considera o cliente como rebelde, agitado, confuso, quando, na verdade, ele pode estar apresentando dificuldades de adaptação ao ambiente, que não é o dele ou por estar junto de pessoas com as quais não convive. Assim, é importante que a equipe, que atende o cliente e sua família, atente para o que está velado ou oculto por trás das queixas, rebeldias e agitações, devendo considerar o apoio psicológico, como um recurso a mais a ser utilizado para o entendimento do contexto situacional ${ }^{(20)}$.

Acrescenta-se, nos resultados, o quanto que a abordagem psicológica em saúde precisa considerar simultaneamente o sujeito, a família, os técnicos de saúde uma vez que o ambiente hospitalar, por si só, representa um grande gerador de desgaste de vários tipos e em diferentes níveis e requerem atenção ${ }^{(21)}$.
Apesar do esgotamento físico e mental profissional ser uma realidade em estudos no âmbito hospitalar ${ }^{(21)}$ os familiares nesse estudo reconheceram que os profissionais possuem características que os agradam e reforçam o quão desejam que eles continuem sendo o que são.

\section{CONCLUSÃO}

A percepção dos familiares que viveram a experiência de serem acompanhantes de clientes internados nas unidades de clínica médica e cirúrgica em relação aos sentimentos pela equipe de enfermagem foi considerada positiva e satisfatória. Metade dos participantes emitiu sugestões para manter ou melhorar a qualidade da assistência de enfermagem.

Os aspectos técnicos e comportamentais expressos nas ações de enfermagem do cotidiano foram citados como pontos (positivos e negativos) que interferem no relacionamento entre o familiar e a equipe, configurando um foco a ser refletido com atenção pela Enfermagem. Os sentimentos de ser acolhido, receber atendimento pontual e atencioso, provocar bem-estar, ser escutado e percebido foram os mais recordados pelos participantes da pesquisa.

A Enfermagem deve investir cada vez mais no processo de comunicação, uma vez que este subsidiará o cuidado efetivo e eficaz ao cliente hospitalizado. A busca e o resgate de sentimentos, valores e atitudes entre equipe de enfermagem e familiares são os caminhos nos quais se pode alcançar satisfação pela assistência prestada durante o período de tratamento na instituição de saúde.

\section{REFERÊNCIAS}

1. Ferreira MA. A comunicação no cuidado: uma questão fundamental na enfermagem. Rev Bras Enferm. 2006;59(3):327-30.

2. Carneiro TM. Vivenciando o cuidar e o curar como familiar em um hospital. Rev Bras Enferm. 2008;60(3): $391-2$.

3. Salomé GM, Espósito VHC, Moraes ALH. O significado de família para casais homossexuais. Rev Bras Enferm. 2007;60(5):559-63.

4. Casanova EG, Lopes GT. Principais conceitos da abordagem sistêmica em cuidados de enfermagem ao indivíduo e sua família. Rev Bras Enferm. 2009;62(6):831-6. 
5. Squassante ND, Alvim NAT. Relação Equipe de enfermagem e acompanhantes de clientes hospitalizados: implicações para o cuidado. Rev Bras Enferm. 2009;62(1):11-7.

6. Souza Filho AO, Xavier EP, Vieira LJES. Hospitalização na óptica do acidentado de transito e de seu familiar acompanhante. Rev Esc Enferm USP. 2008;42(3):540.

7. Molina RCM, Fonseca EL, Waidman MAP, Marcon SS. A percepção da família sobre sua presença em uma UTI pediátrica e neonatal. Rev Bras Enferm. 2009;43(4):636-6.

8. Bardin L. Análise de conteúdo. $4^{\mathrm{a}}$ ed. Lisboa: Edições 70; 2008 .

9. Silva MJP. O papel da comunicação na humanização da atenção à saúde. Bioética. 2002; 10(2):73-88.

10. Watson J. Assessing and measuring caring in nursing and health sciences. $2^{\mathrm{a}}$ ed. New York: Springer; 2008.

11. Waldow VR. O cuidado na saúde: as relações entre o eu, o outro e o cosmos. Petrópolis: Vozes; 2004.

12. Prochet T, Ruiz T, Correa I. A humanização do atendimento ao idoso: o que o idoso hospitalizado sente, percebe e deseja? Nursing. 2006; 94(9):713-18.

13. Needleman J, Buerhaus P, Stewart M, Zelevinsky K, Soeren M. Nurse staffing in hospitals: is there a business case for quality? Health Affairs. 2006;25(1):204-11.

14. Ainken LH, Clarke SP, Sloane DM, Lake ET, Cheney T. Effects of hospital care environment on patient mortality and nurse outcomes. JONA. 2008; 38(5):223-9

15. Rossetti AC, Gaidzinski RR. Estimativa do quadro de pessoal de enfermagem em um novo hospital. Rev. latino-am. enfermagem 2011;19(4):1011-7

16. Szareski C, Beuter M, Brondani CM. O familiar acompanhante no cuidado ao adulto hospitalizado na visão da equipe de enfermagem. Rev. Gaúcha enferm. 2010;31(4):715-2.

17. Campos LF, Melo MRAC. Dimensionamento de pessoal de enfermagem: parâmetros, facilidades e desafios. Cogitare enferm. 2009;14(2):237-46.

18. Maciel MR, Souza MF. Acompanhante de adulto na unidade de terapia intensiva: uma visão do paciente. Acta Paul. Enferm. 2006;19(2):1-7.
19. Saiote E, Mendes F. A partilha de informação com familiares em unidade de tratamento intensivo: uma importância atribuída por enfermeiros. Cogitare enferm. 2011;16(2):219-25.

20. Barbosa EMA, Brasil VV. Boletim informativo em UTI: percepção de familiares e profissionais de saúde. Rev. Eletr. Enf. [Internet] 2007;9(2):315-28.

21. Nascimento KC, Erdmann AL. Understanding the dimensions of intensive care: transpersonal caring and complexity theories. Rev. Latino-Am. Enfermagem. 2009;17(2):215-21. 